Received: 31 January 2017

Accepted: 12 July 2017

Published online: 15 August 2017

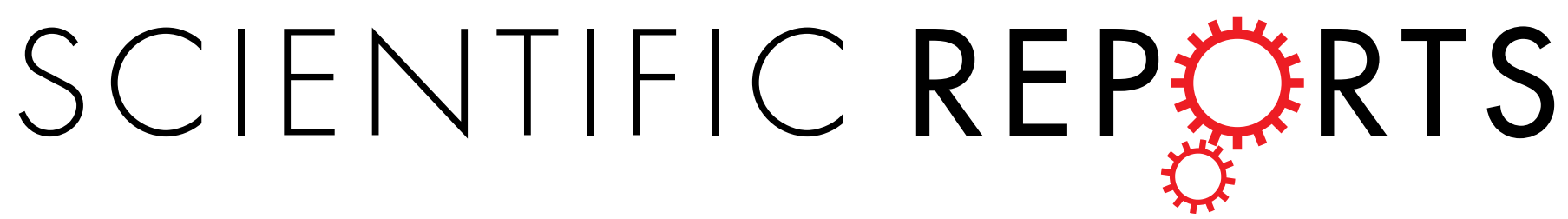

\title{
OPEN Octahedron Iron Oxide Nanocrystals Prohibited Clostridium difficile Spore Germination and Attenuated Local
and Systemic Inflammation
}

Wei-Ting Lee ${ }^{1}$, Ya-Na Wu ${ }^{2}$, Yi-Hsuan Chen ${ }^{3}$, Shang-Rung Wu' ${ }^{2}$, Tsai-Miao Shih ${ }^{2}$, Tsung-Ju Li ${ }^{1}$, Li-Xing Yang ${ }^{1}$, Chen-Sheng Yeh ${ }^{4}$, Pei-Jane Tsai ${ }^{1,3,5}$ \& Dar-Bin Shieh ${ }^{1,2,6,7}$

Clinical management of Clostridium difficile infection is still far from satisfactory as bacterial spores are resistant to many chemical agents and physical treatments. Certain types of nanoparticles have been demonstrated to exhibit anti-microbial efficacy even in multi-drug resistance bacteria. However, most of these studies failed to show biocompatibility to the mammalian host cells and no study has revealed in vivo efficacy in C. difficile infection animal models. The spores treated with $500 \mu \mathrm{g} / \mathrm{mL} \mathrm{Fe}{ }_{3-8} \mathrm{O}_{4}$ nanoparticles for 20 minutes, $64 \%$ of the spores were inhibited from transforming into vegetative cells, which was close to the results of the sodium hypochlorite-treated positive control. By cryo-electron micro-tomography, we demonstrated that $\mathrm{Fe}_{3-\delta} \mathrm{O}_{4}$ nanoparticles bind on spore surfaces and reduce the dipicolinic acid (DPA) released by the spores. In a C. difficile infection animal model, the inflammatory level triple decreased in mice with colonic $C$. difficile spores treated with $\mathrm{Fe}_{3-\delta} \mathrm{O}_{4}$ nanoparticles.

Histopathological analysis showed a decreased intense neutrophil accumulation in the colon tissue of the $\mathrm{Fe}_{3-\delta} \mathrm{O}_{4}$ nanoparticle-treated mice. $\mathrm{Fe}_{3-\delta} \mathrm{O}_{4}$ nanoparticles, which had no influence on gut microbiota and apparent side effects in vivo, were efficacious inhibitors of $C$. difficile spore germination by attacking its surface and might become clinically feasible for prophylaxis and therapy.

Nanomaterials have attracted significant interest in medicine. Certain microorganism-reactive nanomaterials have been used as alternative bactericides ${ }^{1}$, namely, silver, zinc oxide, and titanium oxide nanoparticles, all of which have remarkable antibacterial properties ${ }^{2,3}$. The antibacterial mechanisms of nanoparticles may be attributable to their generation of reactive oxygen species, disruption of cell membranes, ability to bind thiol groups, and their release of toxic ions ${ }^{4}$. Spore-formation enables bacteria to survive nutritional deprivation and harsh environments. They can resist ultraviolet radiation, desiccation, high temperatures, extreme freezing, and chemical disinfectants ${ }^{5}$. Spores can reactivate themselves to the vegetative state when the environment becomes favorable. Therefore, Clostridium species, spore-forming pathogens, usually challenge clinical disease management and prevention. Clostridium difficile, a pathogen associated with healthcare-facility-related (nosocomial) infections, is a major cause of antibiotic-treatment-related diarrhea, pseudomembranous colitis, abdominal pain, fever, and death ${ }^{6}$. The normal flora in the gut can inhibit the growth of $C$. difficile and therefore protect patients from

\footnotetext{
${ }^{1}$ Institute of Basic Medical Sciences, National Cheng Kung University, 1 University Road, Tainan, 701, Taiwan. ${ }^{2}$ Institute of Oral Medicine, National Cheng Kung University, 1 University Road, Tainan, 701, Taiwan. ${ }^{3}$ Department of Medical Laboratory Science and Biotechnology, National Cheng Kung University, 1 University Road, Tainan, 701, Taiwan. ${ }^{4}$ Department of Chemistry, National Cheng Kung University, 1 University Road, Tainan, 701, Taiwan. ${ }^{5}$ Center of Infectious Disease and Signaling Research, National Cheng Kung University, 1 University Road, Tainan, 701, Taiwan. ${ }^{6}$ Department of Stomatology, National Cheng Kung University Hospital, College of Medicine, National Cheng Kung University, 138 Sheng-Li Road, Tainan, 704, Taiwan. ${ }^{7}$ Advanced Optoelectronic Technology Center and Center for Micro/Nano Science and Technology, National Cheng Kung University, 1 University Road, Tainan, 701, Taiwan. Correspondence and requests for materials should be addressed to P.-J.T. (email: peijtsai@mail.ncku.edu. tw) or D.-B.S. (email: dbshieh@mail.ncku.edu.tw)
} 


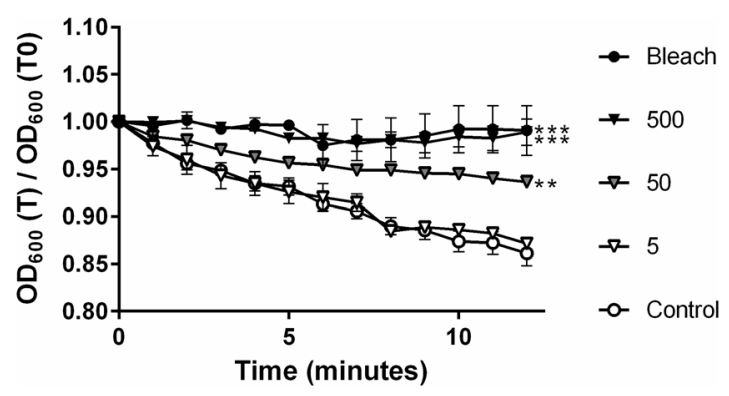

Figure 1. C. difficile spore germination was significantly inhibited in spores treated with $\mathrm{Fe}_{3-\delta} \mathrm{O}_{4}$ nanoparticles. Purified CCUG 37780 spores were incubated in BHIS medium containing $\mathrm{Fe}_{3-8} \mathrm{O}_{4}$ nanoparticles ( $[500 \mu \mathrm{g} / \mathrm{mL}$ $(\Delta), 50 \mu \mathrm{g} / \mathrm{mL}(\Delta)$, or $5 \mu \mathrm{g} / \mathrm{mL}(\Delta)])$, or $3 \%$ sodium hypochlorite as a positive control. The kinetics of spore germination was analyzed using spectrometric absorption referenced to the starting point. $\mathrm{OD}_{600}(\mathrm{~T})=$ different time points after taurocholate treatment; $\mathrm{OD}_{600}\left(\mathrm{~T}_{0}\right)=$ time zero. Spore germination was significantly inhibited in spores treated with $\mathrm{Fe}_{3-\delta} \mathrm{O}_{4}$ nanoparticles. (*** $\mathrm{P}<0.001$; one-way analysis of variance [ANOVA] followed by Tukey's Multiple Comparison test).

developing C. difficile infection ${ }^{7}$. C. difficile infection usually occurs in patients on a long-term regimen of antibiotics, and it is often initiated by the spores acquired from healthcare workers, ${ }^{8,9}$. Once a patient develops C. difficile infection, there are only a few antibiotics available to control it ${ }^{10}$. Moreover, the failure rate of first-line antibiotics and the $C$. difficile infection relapse rate are both dramatically high ${ }^{10,11}$. Consequently, about two decades ago, the attributable post-diagnosis mortality rate was $6.9 \%$ at 30 days and $16.7 \%$ at 1 year ${ }^{12}$. The spores of C. difficile are the major cause of $C$. difficile infection. Compared with oxygen-sensitive vegetative bacteria, $C$. difficile spores survive for up to several months in room air and in low- $\mathrm{pH}$ gastric contents ${ }^{13}$. As the spores enter the human digestive tract, they germinate after they have been exposed to bile salts and their derivatives, and then they are colonized in the colon ${ }^{14}$. The virulence of $C$. difficile depends upon the gene expression of $t c d \mathrm{~A}$-encoded toxin $\mathrm{A}$, an enterotoxin, and $t c d \mathrm{~B}$-encoded toxin $\mathrm{B}$, a cytotoxin ${ }^{15}$. Both cause intestinal inflammation and neutrophil infiltration in the infected foci ${ }^{16,17}$.

The incidence of $C$. difficile infection has significantly increased in the past 15 years ${ }^{18}$. C. difficile infection has become a major cause of nosocomial-associated infection in the world ${ }^{9}$. Antibiotic-resistant $C$. difficile is not only potentially fatal, but it also causes healthcare-associated economic burdens ${ }^{19}$. The available present antibiotics are targeted to vegetative bacterium, however, the infective form is the spore. Current $C$. difficile infection clinical management is still far from satisfactory because the spores are resistant to many chemical agents and physical treatments, which makes effective management of the spores an important problem ${ }^{20}$. Therefore, anti-germination approach could lead to the prevention of $C$. difficile infection. Some newly designed cholate derivatives show promise against $C$. difficile infection; however, they are still under pre-clinical study ${ }^{21,22}$. Sodium hypochlorite, a standard disinfectant, has outstanding antimicrobial activity but undesirable side effects: it is corrosive and irritates tissue ${ }^{5}$. To control spore germination and C. difficile infection, it is important to develop an efficacious and biocompatible spore-control strategy.

There are various well-known antibacterial nanomaterials, e.g., silver ( $\mathrm{Ag})$ and zinc oxide $(\mathrm{ZnO})$ nanoparticles $^{23,24}$, and zero-valent iron nanoparticles, which are prominently bactericidal against Escherichia coli ${ }^{25}$. The nanotechnology even has been reported that it could overcome the problem of multi-drug resistant bacteria ${ }^{26}$. However, most current antibacterial nanomaterials are primarily multifunctional generic biocidal agents against vegetative cells; their sporicidal activity at high concentrations has been explored in only a few studies ${ }^{27,28}$. Many studies demonstrated the excellent antibacterial ability of nanoparticles, but their impacts to the gut microbiota were not clear. The healthy gut microbiota prevent the host from colonic pathogen ${ }^{29}$. Therefore, an ideal antibacterial nanomaterial should exhibit the specific pathogen targeting capability without causing disruption of healthy gut microibota.

The toxicity of various bactericidal nanoparticles to mammalian cells has become an increasing concern. Iron-containing particles are generally recognized as highly biocompatible nanomaterials. A few specific iron oxide nanoparticles have recently been reported to be antibacterial; they were synthesized and showed therapeutic potential for cancer therapy ${ }^{30,31}$. In this study, we evaluated whether such single-crystal nonstoichiometric $\mathrm{Fe}_{3-\delta} \mathrm{O}_{4}$ magnetite nanoparticles could inhibit C. difficile spore germination in vivo. The potential mechanisms were explored from both material science and molecular biology perspectives.

\section{Results}

Testing the sporicidal activity of $\mathrm{Fe}_{3-\delta} \mathrm{O}_{4}$ nanoparticles against $C$. difficile. We used a previously described method ${ }^{32}$ to explore the inhibitory efficacy of $\mathrm{Fe}_{3-\delta} \mathrm{O}_{4}$ nanoparticles to spores. After they had been incubated with sodium hypochlorite for 20 minutes, inactivated spores were used as positive controls, as described elsewhere ${ }^{33}$. After they had been incubated with nanomaterials of various concentrations $(5,50$, and $500 \mu \mathrm{g} / \mathrm{mL})$ for 20 minutes, the treated spores were then stimulated using $10 \mathrm{mM}$ taurocholate to induce germination.

Spores treated with 50 and $500 \mu \mathrm{g} / \mathrm{mL}$ of $\mathrm{Fe}_{3-\delta} \mathrm{O}_{4}$ nanoparticles showed significantly $(* * \mathrm{P}<0.01$ and $* * * \mathrm{P}<0.001$ ) inhibited germination (Fig. 1), but spores treated with $5 \mu \mathrm{g} / \mathrm{mL}$ did not. There were no significant differences between the sodium hypochlorite-treated positive controls and the $\mathrm{Fe}_{3-\delta} \mathrm{O}_{4}$ nanoparticle-treated 
(A)

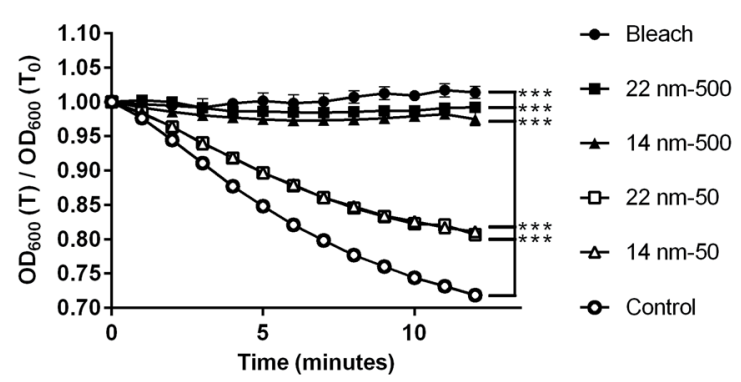

(B)

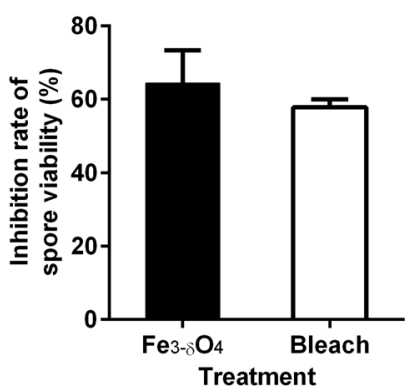

(C)

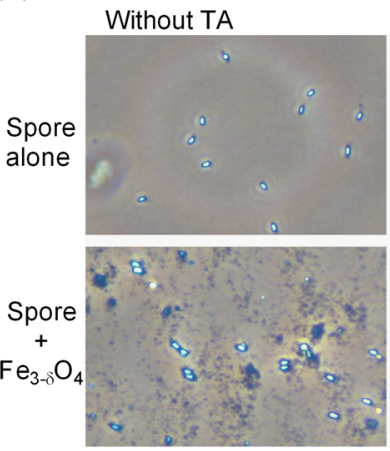

With TA

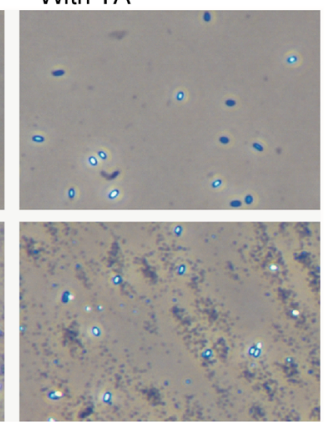

(D)

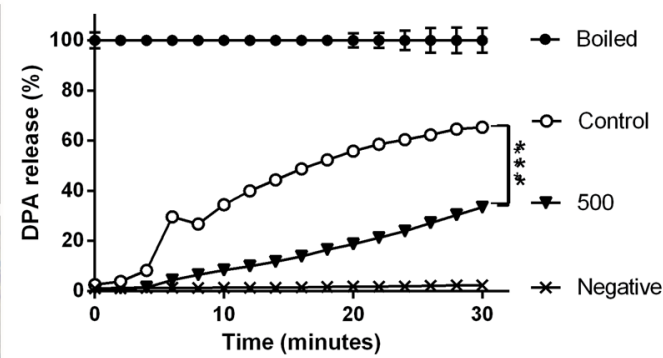

Figure 2. The viability and germination inhibition of C. difficile CCUG 37780 spores by $\mathrm{Fe}_{3-\delta} \mathrm{O}_{4}$ nanoparticles were dose-dependent rather than size-dependent. (A) The spores were first treated for 20 minutes with $500 \mu \mathrm{g} / \mathrm{mL}$ of $22-\mathrm{nm} \mathrm{Fe}_{3-\delta} \mathrm{O}_{4}(\boldsymbol{\square}), 500 \mu \mathrm{g} / \mathrm{mL}$ of $14-\mathrm{nm} \mathrm{Fe}_{3-\delta} \mathrm{O}_{4}(\boldsymbol{\Delta}), 50 \mu \mathrm{g} / \mathrm{mL}$ of $22-\mathrm{nm} \mathrm{Fe}_{3-\delta} \mathrm{O}_{4}(\square), 50 \mu \mathrm{g} /$ $\mathrm{mL}$ of $14-\mathrm{nm} \mathrm{Fe}_{3-\delta} \mathrm{O}_{4}(\Delta)$, or $3 \%$ sodium hypochlorite $(\mathbf{)})$ and then were treated with taurocholate to induce germination. Both 14-nm and 22-nm $\mathrm{Fe}_{3-8} \mathrm{O}_{4}$ nanoparticles had a similar dose-dependent effect on spore germination. (B) After C. difficile spores and $500 \mu \mathrm{g} / \mathrm{mL}$ of $22-\mathrm{nm} \mathrm{Fe}_{3-8} \mathrm{O}_{4}$ nanoparticles or $3 \%$ sodium hypochlorite had been incubated for 20 minutes, the spores were plated on BHIS agar for a colony formation assay the next day. The level of colony-forming unit inhibition was similar for $\mathrm{Fe}_{3-\delta} \mathrm{O}_{4}$ nanoparticle- and sodium hypochlorite-treated spores. (C) The spores were treated for 20 minutes with $500 \mu \mathrm{g} / \mathrm{mL}$ of $22-\mathrm{nm} \mathrm{Fe}_{3-\delta} \mathrm{O}_{4}$ and then stimulated using $10 \mathrm{mM}$ taurocholate. After 15 minutes, the phase contrast of the spores was recorded under a phase contrast microscope. (D) The DPA-release assay showed that $\mathrm{Fe}_{3-\delta} \mathrm{O}_{4}$ nanoparticles-treated spores released less DPA than did the control group. Data are mean $\pm \mathrm{SEM}$. (***P $<0.001$; one-way analysis of variance (ANOVA) followed by Tukey's Multiple Comparison test).

$(500 \mu \mathrm{g} / \mathrm{mL})$ spores (Fig. 1). Intriguingly, there was no significant difference in germination inhibition between spores treated with 14-nm and 22-nm $\mathrm{Fe}_{3-\delta} \mathrm{O}_{4}$ nanoparticles (Fig. 2A). In Supplementary Table 1, the germination kinetic results show that the Michaelis-Menten constant $(\mathrm{Km})$ for taurocholate-treated C. difficile CCUG 37780 spores increased from 4.34 to $8.43 \mathrm{M}$ in mice treated with $50 \mu \mathrm{g} / \mathrm{mL}$ of $\mathrm{Fe}_{3-\delta} \mathrm{O}_{4}$ nanoparticles. The kinetic analysis suggested that $\mathrm{Fe}_{3-\delta} \mathrm{O}_{4}$ nanoparticles have an inhibiting constant $(\mathrm{Ki})$ of approximately $62 \mu \mathrm{g} / \mathrm{mL}$. To confirm the sporicidal property of $500 \mu \mathrm{g} / \mathrm{mL}$ of $\mathrm{Fe}_{3-\delta} \mathrm{O}_{4}$, the number of $\mathrm{Fe}_{3-\delta} \mathrm{O}_{4}$ - and $3 \%$-sodium hypochlorite-treated colony-forming unit (CFU) spores was counted. Compared with the negative control set, the number of CFUs of $\mathrm{Fe}_{3-8} \mathrm{O}_{4}$-treated and 3\%-sodium hypochlorite-treated spores was $\sim 60 \%$ lower (Fig. $2 \mathrm{~B}$ ). Furthermore, the inhibition rates of CFUs in $\mathrm{Fe}_{3-8} \mathrm{O}_{4}$-treated and in 3\%-sodium hypochlorite-treated spores were not significantly different.

Phase-contrast microscopy showed that in the positive controls, to which taurocholate was added for $15 \mathrm{~min}$ utes, but not in the negative controls, the contrast of the spores transitioned from bright to dark (Fig. 2C). The contrasts of the spores treated with $500 \mu \mathrm{g} / \mathrm{mL} \mathrm{Fe}_{3-8} \mathrm{O}_{4}$ nanoparticles were not significantly different. The luminescence signal that accompanies DPA release was significantly higher during taurocholate-induced spore germination (Fig. 2D). However, the luminescence signal was less intense and less abundant in the $\mathrm{Fe}_{3-8} \mathrm{O}_{4}$-treated group than in the taurocholate-treated group. No luminescence signal was detected in the group of spores not treated with taurocholate because the spores did not release DPA.

Inhibitory effect of $\mathrm{Fe}_{3-\delta} \mathrm{O}_{4}$ nanoparticles on virulent strains of $C$. difficile. C. difficile strain CCUG 37780 does not have the $t c d A$ and $t c d B$ genes ${ }^{34}$. To determine what doses of $\mathrm{Fe}_{3-\delta} \mathrm{O}_{4}$ nanoparticles affect the spore germination of $C$. difficile, CCUG 19126 and BAA-1805 tcdA- and $t c d B$-positive strains were treated for 20 minutes with 5,50 , and $500 \mu \mathrm{g} / \mathrm{mL}$ doses of $\mathrm{Fe}_{3-8} \mathrm{O}_{4}$ nanoparticles, and then treated with $10 \mathrm{mM}$ taurocholate. The germination of $C$. difficile CCUG 19126 spores and of $C$. difficile BAA-1805 spores was not significantly different 
(A) C. difficile CCUG 19126

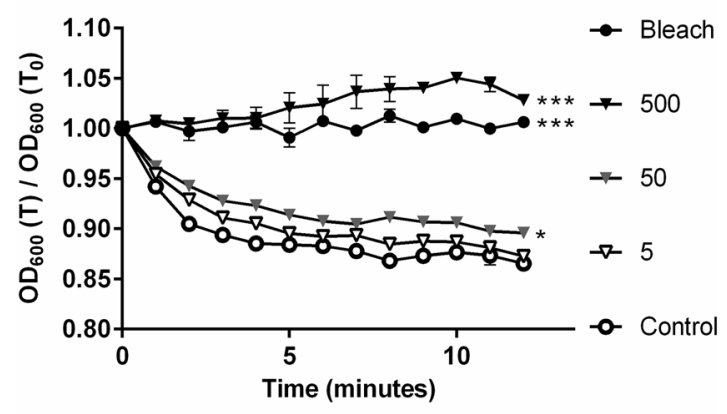

(B) C. difficile ATCC BAA-1805

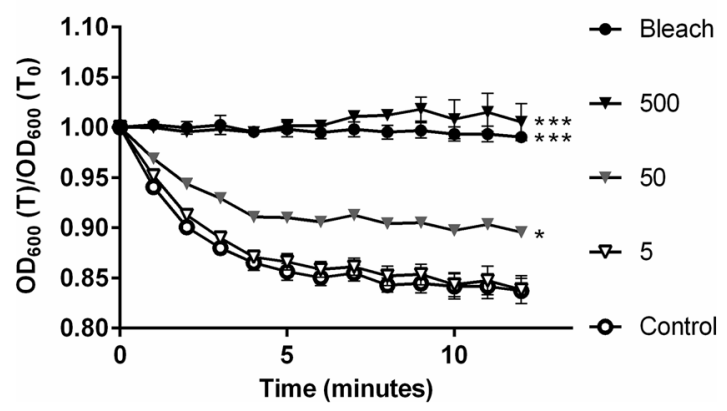

Figure 3. Spore germination was significantly inhibited in $\mathrm{Fe}_{3-8} \mathrm{O}_{4}$-treated pathogenic strains of C. difficile. Spores from two pathogenic strains of $C$. difficile (CCUG 19126 and ATCC BAA-1805) were treated for 20 minutes with various concentrations of $\mathrm{Fe}_{3-\delta} \mathrm{O}_{4}$ nanoparticles and then with $10 \mathrm{mM}$ taurocholate to induce spore germination. Spore germination was effectively inhibited in the two $\mathrm{Fe}_{3-8} \mathrm{O}_{4}$-treated pathogenic strains, as it was in the nonpathogenic CCUG 37780 strain. ( $* * * \mathrm{P}<0.001, * \mathrm{P}<0.05$; one-way analysis of variance [ANOVA] followed by Tukey's Multiple Comparison test).

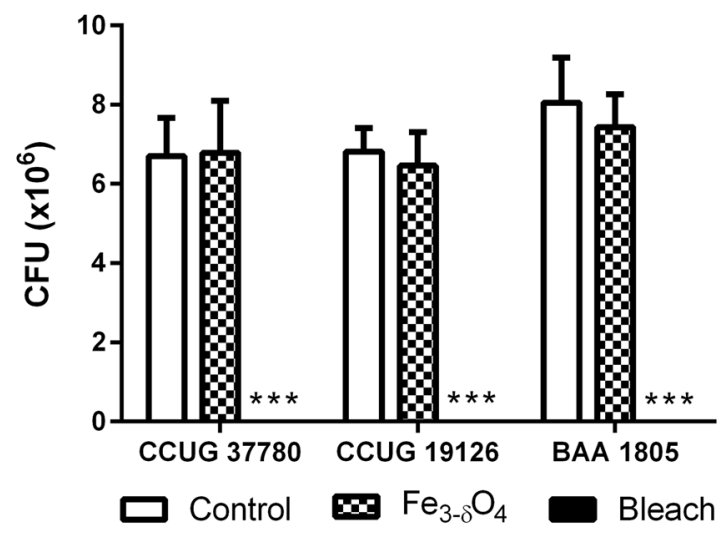

Figure 4. The viability of $C$. difficile vegetative cells was not significantly affected by $\mathrm{Fe}_{3-\delta} \mathrm{O}_{4}$-treatment. $\mathrm{Fe}_{3-\delta} \mathrm{O}_{4}$ nanoparticles $(500 \mu \mathrm{g} / \mathrm{mL})$ did not inhibit the viability of vegetative C. difficile in all three test strains compared cells treated with $3 \%$ sodium hypochlorite. $(* * * \mathrm{P}<0.001$; Student's $t$ test).

between those treated with $500 \mu \mathrm{g} / \mathrm{ml}$ of $\mathrm{Fe}_{3-\delta} \mathrm{O}_{4}$ nanoparticles and those treated with sodium hypochlorite, but CCUG 19126 was relevantly resistant to treatment with $50 \mu \mathrm{g} / \mathrm{mL}$ of $\mathrm{Fe}_{3-\delta} \mathrm{O}_{4}$ nanoparticles (Fig. $3 \mathrm{~A}$ ).

To evaluate the bactericidal effect of $\mathrm{Fe}_{3-\delta} \mathrm{O}_{4}$ nanoparticles on vegetative cells in different strains, three groups of vegetative cells-CCUG 37780, CCUG 19126, and BAA-1805-were first treated for 20 minutes with $500 \mu \mathrm{g} /$ $\mathrm{mL}$ of $\mathrm{Fe}_{3-\delta} \mathrm{O}_{4}$ nanoparticles, spread on BHIS agar plates, and their CFUs were counted. The growth behavior of the $\mathrm{Fe}_{3-\delta} \mathrm{O}_{4}$-treated vegetative cells was not significantly different from that of the negative control groups; however, almost all the vegetative cells treated with $3 \%$ sodium hypochlorite died (Fig. 4). Treatment with $\mathrm{Fe}_{3-\delta} \mathrm{O}_{4}$ nanoparticles did not damage $C$. difficile vegetative cells. 
(A)

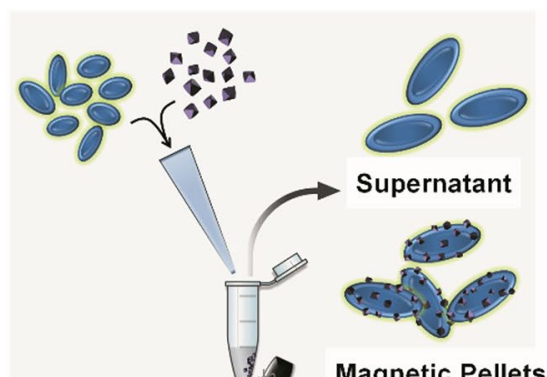

Magnetic Pellets
(B)

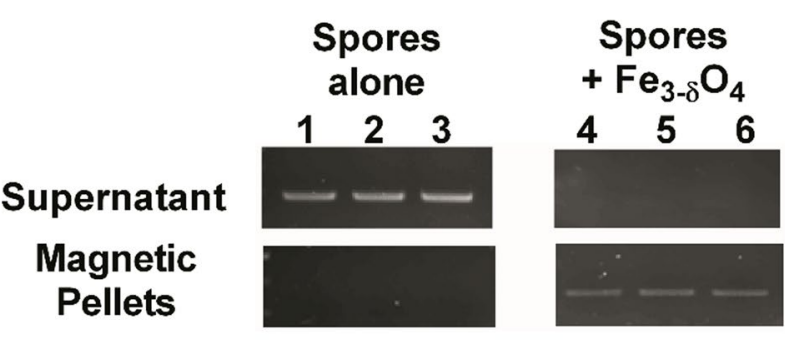

(C)
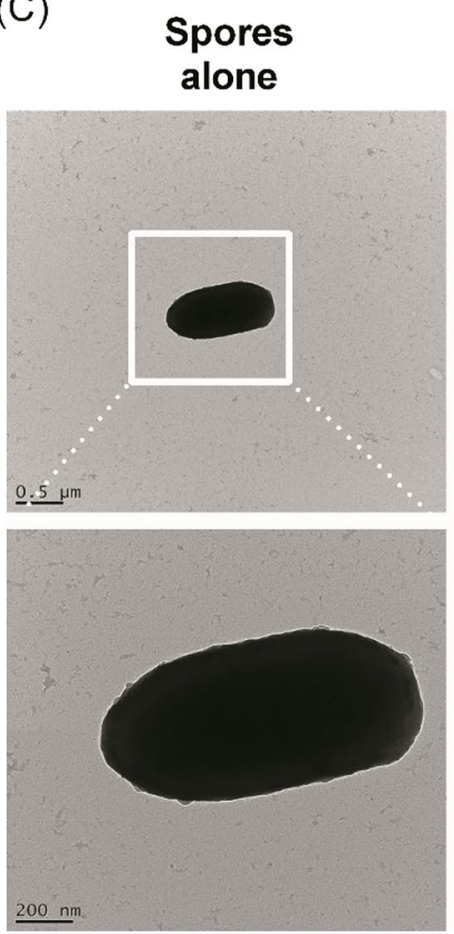
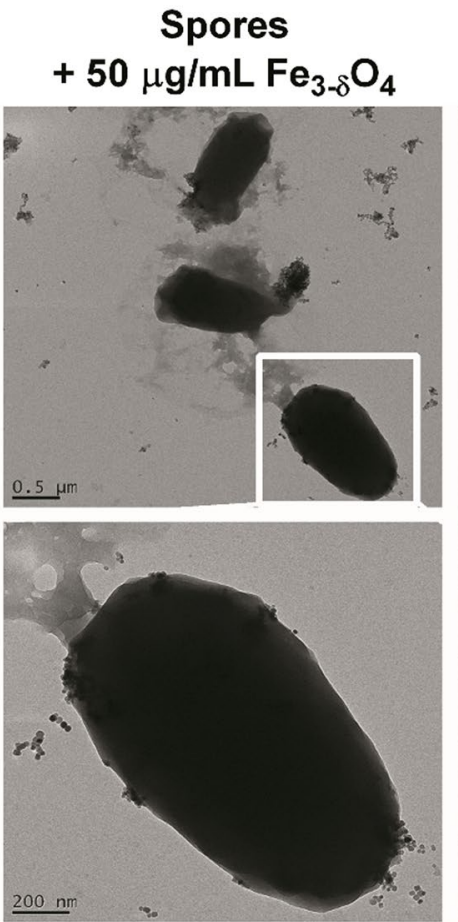
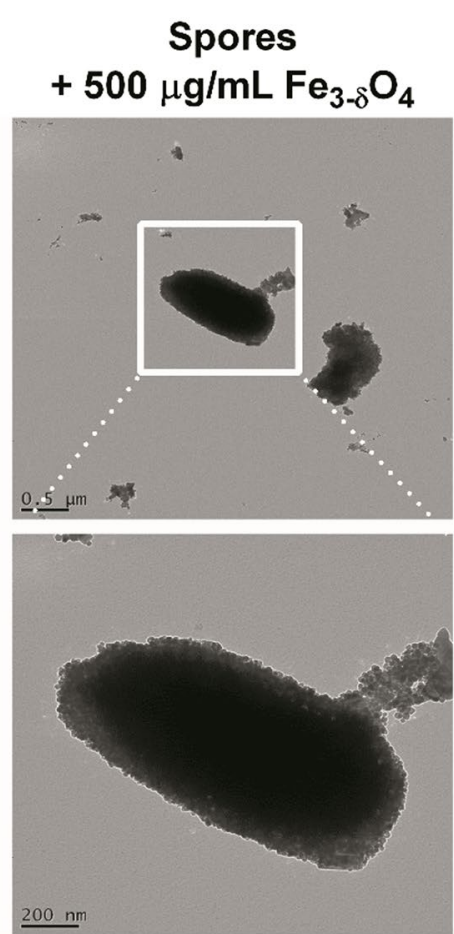

Figure 5. $\mathrm{Fe}_{3-\delta} \mathrm{O}_{4}$ nanoparticles bound to the surface of $C$. difficile (ATCC BAA-1805) spores, which allowed magnetic attraction. (A) Schematic illustration of the magnetic separation of spores with which $\mathrm{Fe}_{3-\delta} \mathrm{O}_{4}$ nanoparticles had bound: these spores were attracted by a magnet, but spores with which the nanoparticles had not bound were retained in the supernatant. (B) The spores were incubated for 20 minutes with $\mathrm{Fe}_{3-\delta} \mathrm{O}_{4}$ nanoparticles, and then $t c d B$ genes were isolated from the supernatant or the magnetically concentrated precipitates were detected after PCR amplification. Lanes 1-3 are spores alone and lanes 4-6 are spores treated with $500 \mu \mathrm{g} / \mathrm{mL}$ of $\mathrm{Fe}_{3-\delta} \mathrm{O}_{4}$ nanoparticles. TcdB amplicons were detected in the supernatant of the control group, but they were found only in the magnetically concentrated fraction after they had been treated with $\mathrm{Fe}_{3-\delta} \mathrm{O}_{4}$ nanoparticles. (C) TEM images of the spores (upper panel: 10,000 $\times$; lower panel: $30,000 \times$ ). The left image shows that native spores have a smooth coat; accumulated $\mathrm{Fe}_{3-\delta} \mathrm{O}_{4}$ nanoparticles on spore surfaces are evident after incubation. The spores treated with $500 \mu \mathrm{g} / \mathrm{mL}$ were completely covered by the $\mathrm{Fe}_{3-\delta} \mathrm{O}_{4}$ nanoparticles.

The mechanisms underlying $\mathrm{Fe}_{3-\delta} \mathrm{O}_{4}$-inhibited spore germination. To determine whether any additional underlying mechanisms allow $\mathrm{Fe}_{3-\delta} \mathrm{O}_{4}$ nanoparticles to inhibit spore germination, we first excluded the sporicidal activity of the coating polymer poly-(styrene-alt-maleic acid) (PSMA) on $\mathrm{Fe}_{3-\delta} \mathrm{O}_{4}$ nanoparticles. At a concentration higher than that on PSMA-coated on $\mathrm{Fe}_{3-\delta} \mathrm{O}_{4}$ nanoparticles, PSMA did not inhibit spore germination (Supplementary Fig. 1). In addition, to verify whether $\mathrm{Fe}_{3-8} \mathrm{O}_{4}$ nanoparticles promoted or hindered the germination-inducing activity of taurocholate, $10 \mathrm{mM}$ taurocholate was incubated for 20 minutes with different concentrations of $\mathrm{Fe}_{3-\delta} \mathrm{O}_{4}$ nanoparticles and then used to treat spores. The taurocholate incubated with $\mathrm{Fe}_{3-\delta} \mathrm{O}_{4}$ nanoparticles still induced C. difficile spore germination (Supplementary Fig. 2).

To identify any additional underlying mechanisms that allow $\mathrm{Fe}_{3-\delta} \mathrm{O}_{4}$ nanoparticles to inhibit spore germination, we took advantage of the magnetic property of $\mathrm{Fe}_{3-\delta} \mathrm{O}_{4}$. Some $C$. difficile BAA-1805 spores were incubated 
with $500 \mu \mathrm{g} / \mathrm{mL}$ of $\mathrm{Fe}_{3-\delta} \mathrm{O}_{4}$ nanoparticles for 20 minutes and then separated, using a magnet, into two parts: the nonmagnetic supernatant and the magnetic pellets (Fig. 5A). Each part was collected, and PCR was used to detect the $t c d B$ gene. The $t c d B$ gene was detected only in the supernatant (Fig. 5B) in the spores-alone groups. In the $\mathrm{Fe}_{3-8} \mathrm{O}_{4}$-treated spores, the $t c d B$ gene was also detected in the magnetic pellets.

To clarify the interaction between $\mathrm{Fe}_{3-\delta} \mathrm{O}_{4}$ and spores, the $\mathrm{Fe}_{3-\delta} \mathrm{O}_{4}$-treated spores were examined under a TEM. The TEM images showed that spores treated with $50 \mu \mathrm{g} / \mathrm{mL}$ of $\mathrm{Fe}_{3-\delta} \mathrm{O}_{4}$ nanoparticles were only spotted with $\mathrm{Fe}_{3-\delta} \mathrm{O}_{4}$ nanoparticles, but that spores treated with $500 \mu \mathrm{g} / \mathrm{mL}$ were almost fully surrounded (Fig. $5 \mathrm{C}$ ). To reconfirm that $\mathrm{Fe}_{3-\delta} \mathrm{O}_{4}$ nanoparticles were simply attached to the surface of the spores instead of diffused into the spores, the spores were examined using cryo-electron tomography. The rotated dynamic video (Supplementary Video 1) revealed that $\mathrm{Fe}_{3-\delta} \mathrm{O}_{4}$ nanoparticles were directly and dose-dependently bound to the surface of $C$. difficile spores.

$\mathrm{Fe}_{3-\delta} \mathrm{O}_{4}$ nanoparticles as an in vivo sporicidal agent against $C$. difficile infection. Before the mouse experiments, the cytotoxicity of $\mathrm{Fe}_{3-\delta} \mathrm{O}_{4}$ nanoparticles was evaluated using a methylthiazol tetrazolium (MTT) assay. The colorectal cells were treated with various concentrations of $\mathrm{Fe}_{3-\delta} \mathrm{O}_{4}$ nanoparticles $(5$ to $500 \mu \mathrm{g} /$ $\mathrm{mL}$ ) for 24 hours. The viability of C2BBe1 cell line were decreased at the $500 \mu \mathrm{g} / \mathrm{mL}$ of $\mathrm{Fe}_{3-\delta} \mathrm{O}_{4}$ nanoparticles. However, the cell viability assay showed no significant cytotoxicity of the $\mathrm{Fe}_{3-\delta} \mathrm{O}_{4}$ nanoparticles in HT-29 cell line compared with the control group (Supplementary Fig. 3). To investigate the feasibility of $\mathrm{Fe}_{3-8} \mathrm{O}_{4}$ nanoparticles as an in vivo sporicidal agent, we used a $C$. difficile infection mouse model. The inflammatory signal peaked 3 days after C. difficile infection had been induced (Supplementary Fig. 4). Therefore, in the mouse experiment, we examined the $C$. difficile infection pathology 3 days after the mice had been treated with spores. Mice treated with $\mathrm{Fe}_{3-\delta} \mathrm{O}_{4}$ nanoparticles plus spores lost significantly less body weight than did the mice treated with spores alone $(\mathrm{P}=0.0119$, Student's $t$ test $)$ (Fig. 6A). The cecum weight of the $\mathrm{Fe}_{3-\delta} \mathrm{O}_{4}$-treated mice was significantly higher than that of the mice treated with spores alone ( $\mathrm{P}=0.0024$, Student's $t$ test) (Fig. $6 \mathrm{~B})$, which showed minor inflammation under an in vivo imager ( $\mathrm{P}=0.0406$, Student's $t$ test) (Fig. 6C). The histopathological images also showed that neutrophil infiltration was lower in the mice treated with $\mathrm{Fe}_{3-\delta} \mathrm{O}_{4}$ nanoparticles plus spores (Fig. 6D) than in the control mice treated with spores alone. A real-time reverse transcription quantitative polymerase chain reaction (RT-qPCR) was used to determine the expression of the proinflammatory genes TNF- $\alpha$, IFN- $\gamma$, and IL-1 $\beta$, compared with the spores-alone groups (Fig. 6E). The level of inflammation caused by the spores was significantly lower $(\mathrm{P}=0.0088$ for TNF- $\alpha, P=0.0276$ for IFN- $\gamma$, and $\mathrm{P}=0.0097$ for IL- $1 \beta$, Student's $t$ test $)$ at the molecular level in the mice treated with $\mathrm{Fe}_{3-\delta} \mathrm{O}_{4}$ nanoparticles plus spores than in the mice treated with spores alone. To verify whether treatment with $500 \mu \mathrm{g} / \mathrm{mL} \mathrm{Fe}_{3-8} \mathrm{O}_{4}$ nanoparticles alone induces any adverse side effects in vivo, we fed the mice with nanoparticles alone and monitored the biochemistry markers (Supplementary Fig. 5). Based on the ratio of Firmicutes to Bacteroidetes, we found this nanoparticle does not altered the gut microbiota (Supplementary Fig. 6A), suggesting that this particle did not has the toxicity to the mice.

\section{Discussion}

Based on our review of the literature, this is the first study which shows Fe-based nanoparticles that strongly inhibited the germination of $C$. difficile spores despite the growth inhibitory effect of other type of iron oxide nanoparticles to other bacteria vegetative cells was recently reported.

Iron oxide nanoparticles have existed in the earth's stratum and some organisms ${ }^{35}$. Due to the iron oxide nanoparticles are widely used in medical applications. The iron oxide nanoparticles not only can be the MRI T2 contrast material but also be conjugated to other dyes as detection reagents for different imaging systems ${ }^{35}$. The iron oxide nanoparticles could be used as hyperthermia-based cancer therapeutic reagent when combined to radiofrequency. The iron oxide nanoparticles could even be modified with different materials to become the multi-functional nanoparticles, e.g., targeting, drug releasing, and imaging abilities ${ }^{36}$. We performed the new function of iron oxide nanoparticles that could be a C. difficile spore germination inhibition reagent in the study. The 22-nm- $\mathrm{Fe}_{3-\delta} \mathrm{O}_{4}$ nanoparticles presented as unique octahedron single crystals, and the ratio of iron to oxide was 2.966:4, similar to our previously reported 6-nm $\mathrm{Fe}_{3} \mathrm{O}_{4}$ nanoparticles ${ }^{30}$. The differences between $\mathrm{Fe}_{3-\delta} \mathrm{O}_{4}$ and $\mathrm{Fe}_{3} \mathrm{O}_{4}$ include size, surface chemistry, and the presence of minor non-oxidized Fe voids in the $\mathrm{Fe}_{3-\delta} \mathrm{O}_{4}$ particles. There was no significant difference between the inhibitory ability of the 22-nm and the $14-\mathrm{nm} \mathrm{Fe}_{3-\delta} \mathrm{O}_{4}$ doses. The amount of DPA released from the spores was significantly lower in the $\mathrm{Fe}_{3-\delta} \mathrm{O}_{4}$-treated group than in the positive controls. The transition of spores from bright to dark can be observed with a phase-contrast microscope after the DPA has been released ${ }^{37}$. In the present study, because $\mathrm{Fe}_{3-8} \mathrm{O}_{4}$ nanoparticles inhibited spore germination, there was no transition of spores into phase-dark. However, DPA could be detected, but not in as great abundance as with the taurocholate-treated spores, after the spores had been exposed to $\mathrm{Fe}_{3-\delta} \mathrm{O}_{4}$ nanoparticles. Thus, it is conceivable that there is an underlying mechanism that enables $\mathrm{Fe}_{3-\delta} \mathrm{O}_{4}$ nanoparticles both to induce DPA release and to inhibit spore germination. We hypothesized that taurocholate can be caught by $\mathrm{Fe}_{3-8} \mathrm{O}_{4}$ nanoparticles and block the interaction between taurocholate and CspC. CspC is a bile salt germination receptor in C. difficile and transmits the signal to the downstream signaling molecule. However, we later discovered that taurocholate does not bind with $\mathrm{Fe}_{3-\delta} \mathrm{O}_{4}$ nanoparticles, and that taurocholate supernatant without $\mathrm{Fe}_{3-\delta} \mathrm{O}_{4}$ nanoparticles was able to trigger $C$. difficile spore germination. Therefore, we now hypothesize that $\mathrm{Fe}_{3-\delta} \mathrm{O}_{4}$ nanoparticles inhibit bacterial growth without directly interacting with taurocholate. We also hypothesize that $\mathrm{Fe}_{3-\delta} \mathrm{O}_{4}$ nanoparticles do not penetrate spores based on our cryo-TEM Supplementary Video 1. The details of the molecular mechanisms of $\mathrm{Fe}_{3-\delta} \mathrm{O}_{4}$ nanoparticles require additional study.

Zero-valent iron (ZVI) has recently been reported ${ }^{38}$ to be involved in the selective inhibition of cancerous cells while sparing noncancerous cells because it depletes mitochondrial membrane potential. Because mitochondria share distinct structural and biochemical similarities as well as evolutionary links to bacteria, we hypothesize that the nonstoichiometric non-oxidized $\mathrm{Fe}$ of $\mathrm{Fe}_{3-\delta} \mathrm{O}_{4}$ also inhibits spore germination. In fact, one study ${ }^{25}$ reported 
(A)

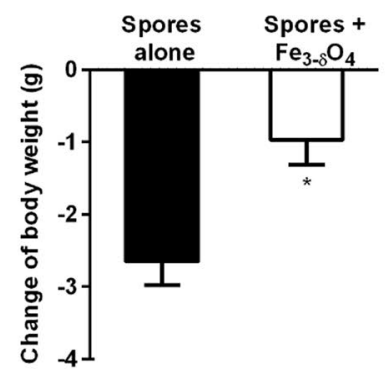

(C)

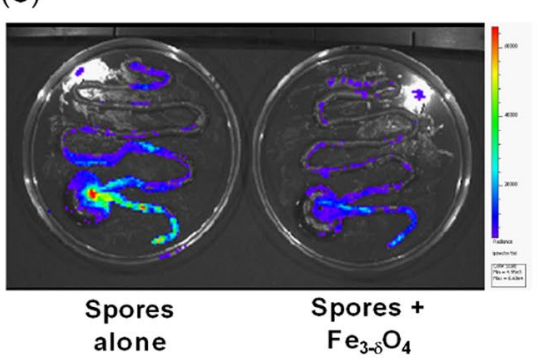

(B)
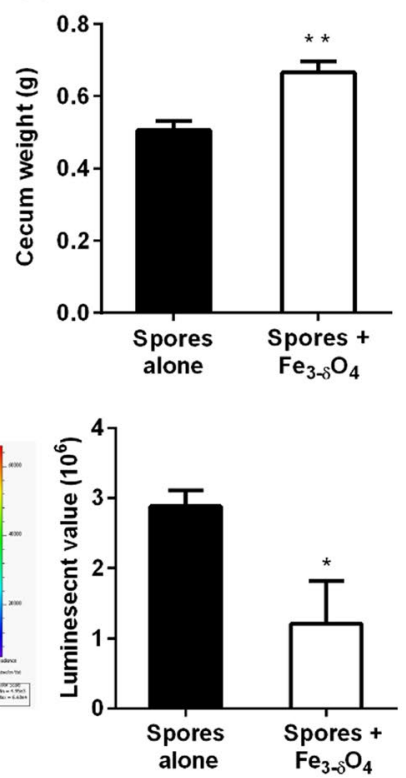

Spores $+\mathrm{Fe}_{3-8} \mathrm{O}_{4}$
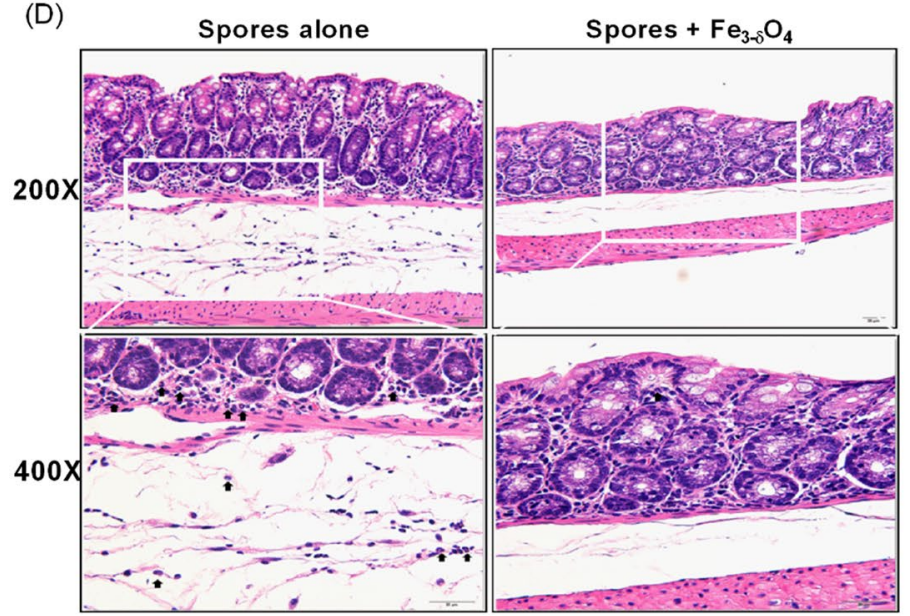

(E)

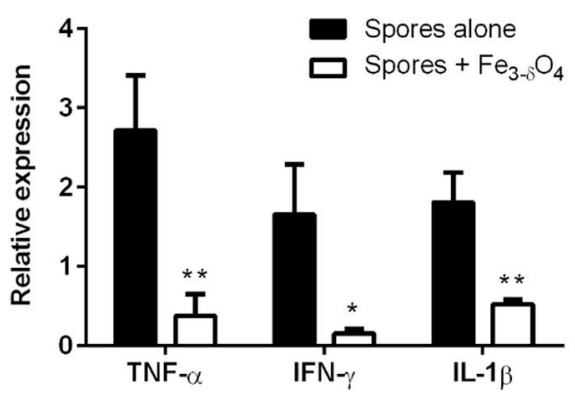

Figure 6. C. difficile infection-induced colitis was attenuated in mice treated with $\mathrm{Fe}_{3-\delta} \mathrm{O}_{4}$ nanoparticles. Purified CCUG 19126 spores were incubated for 20 minutes with $\mathrm{Fe}_{3-\delta} \mathrm{O}_{4}$ nanoparticles, and then the mice were injected with them. Three days later, the mice were killed. (A) The mean bodyweight-loss of the $\mathrm{Fe}_{3-\delta} \mathrm{O}_{4}$ nanoparticle-treated mice was significantly lower than that of the spores-alone control mice. (B) The mean weight-loss of the cecums of the $\mathrm{Fe}_{3-\delta} \mathrm{O}_{4}$ nanoparticle-treated mice was also significantly lower than that of spores-alone control mice. (C) The inflammation of the isolated colon from $11 \mathrm{FNL} / \mathrm{FVB} / \mathrm{NJ}$ transgenic mice with NF- $\kappa \mathrm{B}$-signal-activated bioluminescence was detected. The left image shows a spores-alone control colon and the right image a $\mathrm{Fe}_{3-\delta} \mathrm{O}_{4}$-nanoparticle-treated colon. C. difficile infection-induced inflammation was significantly lower in the $\mathrm{Fe}_{3-\delta} \mathrm{O}_{4}$ nanoparticle-treated colon (right panel). (D) A histopathological examination of the isolated colon tissue shows prominent infiltration of neutrophils (black arrows in left $400 \times$ panel) in the control group, but attenuated inflammation in the $\mathrm{Fe}_{3-8} \mathrm{O}_{4}$ nanoparticle-treated group (right $400 \times$ panel). Neutrophils were morphometrically counted in 10 randomly selected fields in the tissue sections. The number 
of neutrophils in the control group was $16 \pm 5$ per $170 \mathrm{~m} \times 130 \mu \mathrm{m}$ field; the number in the $\mathrm{Fe}_{3-\delta} \mathrm{O}_{4}$ nanoparticles-treated group was $2 \pm 1$ per $170 \mathrm{~m} \times 130 \mu \mathrm{m}$ field. (E) A reverse transcription-quantitative polymerase chain reaction (RT-QPCR) shows that the total RNA extracted from the $\mathrm{Fe}_{3-8} \mathrm{O}_{4}$-nanoparticletreated spores expressed significantly lower levels of proinflammatory genes than did the spores in the control group. $(* \mathrm{P}<0.05, * * \mathrm{P}<0.01$; Student's $t$ test $)(\mathrm{n}=6)$.

a prominent bactericidal effect of ZVI-based nanoparticles on E. coli because they effectively caused oxidative damage to bacterial membranes. Our study showed that $\mathrm{Fe}_{3-\delta} \mathrm{O}_{4}$-treatment significantly inhibited the germination of $C$. difficile spores by damaging spore membranes but not the vegetative cells and therefore did not damage the balance of intestinal normal flora. Thus the specific activity to spore germination must go through other yet to be discovered mechanisms.

It is well known that the expression of the $t c d A$ and $t c d B$ genes is closely associated with the virulence of $C$. difficile infection. We found that $\mathrm{Fe}_{3-\delta} \mathrm{O}_{4}$ nanoparticles inhibited germination in both the $\operatorname{tcd} \mathrm{A}(-) \operatorname{tcdB}(-)$ strain and the more virulent $\operatorname{tcd} \mathrm{A}(+) \operatorname{tcdB}(+)$ strain. In an in vivo $C$. difficile infection mouse experiment, we found that $\mathrm{Fe}_{3-\delta} \mathrm{O}_{4}$ significantly reduced the inflammation indices in the groups treated with spores $+\mathrm{Fe}_{3-\delta} \mathrm{O}_{4}$, and that it effectively inhibited $C$. difficile spore germination, thus controlling in vivo the virulence of $C$. difficile. Intriguingly, treatment with $500 \mu \mathrm{g} / \mathrm{mL}$ of $\mathrm{Fe}_{3-\delta} \mathrm{O}_{4}$ nanoparticles did not kill vegetative cells.

Because antibiotics are overused everywhere in the world, multidrug-resistant microbes have emerged as a major public health threat ${ }^{39}$. Nanomedicines have inspired various new strategies to control microbes without allowing them to develop drug resistance ${ }^{40}$. Bacterial spores are even more resistant than are the bacteria themselves to harsh environments and antibiotics, but nanoparticle-spore interaction and potential clinical applications are rarely discussed. The germination of Bacillus subtilis spores is greatly compromised by treatment with polyionic polymers and then silica nanoparticles ${ }^{41}$. Such treatment, however, does not affect spore viability. We also found that excess PSMA alone did not significantly inhibit the germination of spores; therefore, PSMA must not be important for inhibiting spore germination. In the present study, $500 \mu \mathrm{g} / \mathrm{mL}$ of $\mathrm{Fe}_{3-\delta} \mathrm{O}_{4}$ nanoparticles were sufficient to significantly, and almost as efficaciously as $3 \%$ sodium hypochlorite, inhibit spore germination, which indicated the potential of $\mathrm{Fe}_{3-\delta} \mathrm{O}_{4}$ nanoparticles for clinical development as a novel and effective treatment for managing and perhaps even preventing $C$. difficile infection.

Many studies showed their materials existed excellent antibacterial activity, but sometimes the impact of the materials on the normal flora and biocompatibility were rarely discussed. The guts normal flora plays a vital role in animals, because it attend the metabolism and pathogenic colonization prevention in the host ${ }^{29}$. Therefore, we should have to understand the potential adverse effect of $\mathrm{Fe}_{3-\delta} \mathrm{O}_{4}$ nanoparticles on normal flora and biocompatibility. We found that $\mathrm{Fe}_{3-\delta} \mathrm{O}_{4}$ nanoparticles at a concentration of $500 \mu \mathrm{g} / \mathrm{mL}$ inhibited spore germination but did not damage their vegetative cells. Another study ${ }^{42}$ reported that a high concentration $(3 \mathrm{mg} / \mathrm{mL})$ of $\mathrm{Fe}_{3} \mathrm{O}_{4}$ nanoparticles inhibited the growth of Staphylococcus aureus. After mice were fed with $\mathrm{Fe}_{3-\delta} \mathrm{O}_{4}$ nanoparticles for 72 hours, RT-qPCR was used to analyze the results of normal flora population changes. The two main phyla of bacteria in the gut are Firmicutes and Bacteroidetes ${ }^{43}$. We found that the $\mathrm{Fe}_{3-8} \mathrm{O}_{4}$ nanoparticles did not change the ratio of Firmicutes to Bacteroidetes in the mice (Supplementary Fig. 6A). Bacteroides fragilis and Enterococcus faecalis are the common bacteria in the large intestine ${ }^{44,45}$. Our results showed that $\mathrm{Fe}_{3-\delta} \mathrm{O}_{4}$ nanoparticles did not cause damage to the two bacteria (Supplementary Fig. 6B). According to our results we hypothesize that $\mathrm{Fe}_{3-\delta} \mathrm{O}_{4}$ nanoparticles do not cause imbalance of gut microbiota. The $\mathrm{Fe}_{3-\delta} \mathrm{O}_{4}$ nanoparticles will not be an issue in the normal flora destruction after administering to human and other animals. We also found excellent biocompatibility between $\mathrm{Fe}_{3-\delta} \mathrm{O}_{4}$ nanoparticles and a variety of colorectal cells. We previously reported ${ }^{30,31}$ that $\mathrm{Fe}_{3-\delta} \mathrm{O}_{4}$ nanoparticles used with MRI scans and cancer therapy were safe. Although the safe dosages are different in these three studies, we have shown that $\mathrm{Fe}_{3-\delta} \mathrm{O}_{4}$ nanoparticles are biocompatible and safe in vivo.

Metronidazole and vancomycin are antibiotics frequently used to treat patients with $C$. difficile infection, but the some clinical isolates are metronidazole-resistant ${ }^{46}$. Antibiotics unselectively damage pathogens as well as normal flora and might trigger a highly contagious state of super-shedding $C$. difficile spores, thus increasing the risk of infection ${ }^{47}$. Spores can survive for many years in harsh environments and wait to be ingested by animals ${ }^{48}$. The imbalance of normal flora and spore germination might be the major cause of a C. difficile infection relapse. Moreover, bowel inflammation has long been recognized as an important index for C. difficile infection severity and prognosis. Therefore, we quantitatively assessed C. difficile infection by directly injecting mice with spores, which is most relevant to the pathogenesis of clinical C. difficile infection. Transgenic mice with $\mathrm{NF}-\kappa \mathrm{B}$-dependent luciferase reporter enabled us to visualize bowel inflammation using the IVIS imaging system. Because of their unique ability to selectively kill spores without damaging normal flora, mammalian cells, or the mouse gastrointestinal tract, $\mathrm{Fe}_{3-\delta} \mathrm{O}_{4}$ nanoparticles offer a new strategy for controlling infectious clinical diseases. $\mathrm{Fe}_{3-\delta} \mathrm{O}_{4}$ nanoparticle treatment not only inhibited the germination of different clinical strains of $C$. difficile spores in vitro, but also reduced the virulence of spores that induced $C$. difficile infection in vivo. We also report the first relatively low-dose but efficacious Fe-based nanoparticle inhibition of spore germination, one which uses a novel mechanism that does not interfere with the growth of healthy vegetative flora, which is an important clinical consideration in C. difficile infection management. We hypothesize that coating the surfaces of medical devices with $\mathrm{Fe}_{3-\delta} \mathrm{O}_{4}$ nanoparticles will reduce the threat of spores in hospitals. Additional research on improving the design of $\mathrm{Fe}_{3-8} \mathrm{O}_{4}$ nanoparticles should help nanoparticle-based therapeutics become safer and more effective, and should contribute to the development of new therapeutics for $C$. difficile infection and other emerging infectious diseases. 


\section{Methods}

Ethics statement and animals. All NF- $\mathrm{BB}$-dependent reporter mice (FVB/NJNarl genetic background) were obtained from the National Laboratory Animal Center in Taiwan and housed in a pathogen-free barrier facility. All animal experiments were approved and in accordance with the relevant guidelines and regulations required by the Institutional Animal Care and Use Committee of National Cheng Kung University (NCKU-IACUC-102-296).

Bacterial culture and spore purification. C. difficile CCUG $37780\left(\mathrm{tcdA}^{-}, \mathrm{tcdB}^{-}\right)$and CCUG 19126 $\left(t_{c d A}{ }^{+}, t_{c d B}\right)$ were purchased from the Culture Collections of the University of Göteborg (Göteborg, Sweden), and BAA-1805 $\left(\mathrm{tcdA}^{+}, \mathrm{tcdB}^{+}\right)$from American Type Culture Collection (Manassas, VA). All strains were incubated in brain-heart infusion-supplemented medium (BHIS; BD Difco, Franklin Lakes, NJ), with $0.5 \%$ yeast extract (BD Difco) and $0.1 \%$ L-cysteine (Amresco, Solon, $\mathrm{OH}$ ), at $37^{\circ} \mathrm{C}$ under anaerobic conditions. The spores were prepared and purified as previously described ${ }^{32}$ with a slight modification. Briefly, C. difficile in BHIS medium was diluted in fresh BHIS medium to an optical density (OD) $(600 \mathrm{~nm})$ of 0.2 . The diluted bacterial suspension $(900 \mu \mathrm{L})$ was added to a 6-well dish with BHIS agar, and then the dish was incubated at $37^{\circ} \mathrm{C}$ in an anaerobic jar (Thermo Fisher, Oxoid Ltd., Basingstoke, England) for 4 days. The whole cells were washed 5 times with ice-cold sterile water, the bacteria were resuspended with $3 \mathrm{~mL}$ of ice-cold sterile $\mathrm{MQ}$ water. The suspension was spread on top of a $10-\mathrm{mL} 50 \%$ (wt/vol) sucrose solution (J.T. Baker Chemical, Phillipsburg, PA) in a centrifuge tube, and then centrifuged at $3500 \mathrm{~g}$ for 20 minutes to separate spores from vegetative cells. The purified spores at the bottom of the centrifuge tube were washed 5 times with ice-cold sterile water to remove sucrose, and then stored at $4^{\circ} \mathrm{C}$.

Preparation of the nanoparticles. $\quad \mathrm{Fe}_{3-8} \mathrm{O}_{4}$ nanoparticles $(22 \mathrm{~nm}$ and $14 \mathrm{~nm})$ were synthesized using thermal decomposition, as previously described ${ }^{30}$. Briefly, $1.42 \mathrm{~g}$ of iron acetylacetonate was mixed with $0.57 \mathrm{~mL}$ of oleic acid and $20 \mathrm{~mL}$ of trioctylamine (all 3: Sigma-Aldrich, St. Louis, MO). The solution was refluxed at $325^{\circ} \mathrm{C}$ in an argon environment for 30 minutes. After the solution had cooled down to room temperature, the precipitates were collected with a magnet and washed 3 times with toluene. The $\mathrm{Fe}_{3-8} \mathrm{O}_{4}$ nanoparticles were collected with a magnet and then transferred to chloroform solutions (Merck, Whitehouse Station, NJ) containing $0.4 \mathrm{mg} / \mathrm{mL}$ of PSMA (Sigma-Aldrich) and were left there for 6 hours at $55^{\circ} \mathrm{C}$. The $\mathrm{Fe}_{3-8} \mathrm{O}_{4}$ nanoparticles were collected, washed 3 times with MQ water, and then stored at $4{ }^{\circ} \mathrm{C}$.

Optical density based spore germination test. Spores were germinated as previously described ${ }^{32}$. Before the germination experiments, the spore suspension was incubated at $60^{\circ} \mathrm{C}$ for 30 minutes. The heat-treated spores were then moved to ice. The $C$. difficile spores (concentration: $\mathrm{OD}_{600} 0.5$ ) were co-incubated with various nanoparticles or at different concentrations ( 5 to $500 \mu \mathrm{g} / \mathrm{mL}$ ) in BHIS in a 96-well plate for 20 minutes. The concentration of $\mathrm{Fe}_{3-8} \mathrm{O}_{4}$ nanoparticles was measured based on the total particle weight per $1 \mathrm{~mL}$ of solution. $\mathrm{Fe}_{3-8} \mathrm{O}_{4}(22-\mathrm{nm})$ nanoparticles were used here. Spores treated with 3\% bleach (Wako, Osaka, Japan) were the positive controls to inhibit germination, and spores treated with BHIS only were the negative controls. After the spore + nanoparticle co-incubation, the spores were treated with $10 \mathrm{mM}$ taurocholate (Sigma-Aldrich) to induce germination. The $\mathrm{OD}_{600}$ of treated spores was kinetically determined at 1 minute intervals using a spectrophotometer (TECAN, Grödig, Austria) at room temperature for 12 minutes. The OD at different time points was used to plot the spore germination curve.

Phase-contrast microscopy observation of spore germination. The images of spore germination were recorded using phase-contrast optical microscopy described elsewhere ${ }^{49}$. The purified spores were incubated at $60^{\circ} \mathrm{C}$ for 30 minutes. The spores were co-incubated for 20 minutes with $500 \mu \mathrm{g} / \mathrm{mL}$ of $\mathrm{Fe}_{3-8} \mathrm{O}_{4}$ nanoparticles or with buffer alone. Taurocholate was then added to the samples to a final concentration of $10 \mathrm{mM}$ in BHIS. Five microliters of samples were then dropped onto the $0.7 \%$ agarose surface on the glass slide. After 15 minutes, phase-contrast images of the spores were captured (Nikon Eclipse 80i; Tokyo, Japan). Six random fields of view were obtained for each experimental group.

Dipicolinic acid (DPA) release assay. The DPA release was estimated by modified method from a previous study ${ }^{50}$. Briefly, the spores were heated as described and washed with distilled water then resuspended in spore germination buffer ( $10 \mathrm{mM}$ Tris [pH 7.5], $150 \mathrm{mM} \mathrm{NaCl}$, and $100 \mathrm{mM}$ glycine). The spores were treated with MQ water or $500 \mu \mathrm{g} / \mathrm{mL}$ of $\mathrm{Fe}_{3-8} \mathrm{O}_{4}$ nanoparticles for 20 minutes in a black 96 -well plate before taurocholate $(10 \mathrm{mM})$ and terbium (III) chloride $\left(\mathrm{TbCl}_{3}\right)(100 \mu \mathrm{M})$ were added. The total DPA of the spores was extracted by boiling the samples for 30 minutes. The spores treated with $\mathrm{TbCl}_{3}$ only were negative controls. The DPA- $\mathrm{Tb}$ signal was monitored immediately in real-time using a microplate reader (FlexStation 3 Multi-Mode; Molecular Devices, CA) with excitation/emission at $270 \mathrm{~nm}$ and $545 \mathrm{~nm}$, and a cutoff at $530 \mathrm{~nm}$.

Spore viability analysis. After the spore germination curves had been recorded, each aliquot was removed from the 96-well plate and plated on BHIS agar dishes and serially diluted. The plate-counts (CFU/mL) at each dilution were done after 48 hours of incubation in an anaerobic environment. The inhibition rates were calculated using the following formula:

$$
1:[1-(\text { treatment } \mathrm{CFU} / \text { control CFU) }] \times 100 \% \text {. }
$$


Nanoparticle-spore binding analysis. C. difficile BAA- 1805 spores at a concentration of $\mathrm{OD}_{600} 0.5$ were co-incubated with $\mathrm{Fe}_{3-8} \mathrm{O}_{4}$ nanoparticles $(500 \mu \mathrm{g} / \mathrm{mL})$ in BHIS in a 96-well plate for 20 minutes. The spore samples without $\mathrm{Fe}_{3-\delta} \mathrm{O}_{4}$ treatment were controls. The samples were placed next to a magnet for 5 minutes and then all supernatant was removed to other tubes. The magnet-attracted parts and supernatant were washed 3 times with $1 \times$ phosphate-buffered saline. All samples were resolved in distilled deionized water, and the $t c d B$ DNA in samples was detected using a polymerase chain reaction (PCR). Spore and nanoparticle images were captured using a transmission electron microscope (TEM) (JEM-1400; JEOL, Tokyo, Japan).

In vivo analysis of $\boldsymbol{C}$. difficile infection. To directly monitor the colonic inflammation, we infected mice with $C$. difficile spores in a previously generated NF- $\kappa \mathrm{B}$-dependent reporter mouse model containing the luciferase transgene under the transcriptional control of NF- $\kappa B$ (NF- $\kappa B-R E-l u c i f e r a s e)^{51}$. Before the mice were fed the spores with NF- $\kappa B$ reporter, they were given an antibiotic cocktail $(0.4 \mathrm{mg} / \mathrm{mL}$ of kanamycin, $0.035 \mathrm{mg} / \mathrm{mL}$ of gentamicin, and $0.057 \mathrm{mg} / \mathrm{mL}$ of colistin) in their drinking water for 48 hours and a refreshed cocktail every 24 hours for 2 days. The mice were gavaged with a $200-\mu \mathrm{L}$ proton pump inhibitor (PPI) $(2 \mathrm{mg} / \mathrm{mL})$ every 12 hours for 2 days before the $C$. difficile spore infection. C. difficile CCUG 19126 spores $\left(2 \times 10^{5} \mathrm{CFUs}\right)$ were co-incubated with or without $500 \mu \mathrm{g} / \mathrm{mL}$ of $\mathrm{Fe}_{3-\delta} \mathrm{O}_{4}$ nanoparticles for 20 minutes before they were gavaged with $100 \mathrm{~L}$ of sample solution. While the spores and $\mathrm{Fe}_{3-\delta} \mathrm{O}_{4}$ nanoparticles were incubating, all the mice were gavaged with a $50-\mu \mathrm{L}$ PPI $(2 \mathrm{mg} / \mathrm{mL})$ and then intraperitoneally injected with clindamycin $(4 \mathrm{mg} / \mathrm{kg})$. The antibiotic cocktail water was replaced with normal water after the $C$. difficile infection. All mice were monitored for $C$. difficile infection symptoms, e.g., diarrhea, weight loss, hunched posture, and death. Seventy-two hours post-infection, the mice were intraperitoneally injected with $150 \mathrm{mg} / \mathrm{kg}$ of luciferin (PerkinElmer, Waltham, MA) to show NF- $\kappa B$ activation-mediated luminescence. The mice were anesthetized with isoflurane and oxygen, and then images were collected for 5 minutes using an imaging system (Xenogen IVIS ${ }^{\circledR}$ Spectrum; Advanced Molecular Vision, Grantham, Lincolnshire, UK). Data were analyzed (Xenogen Living Image ${ }^{\circledR}$ ), and luciferase activity was presented in photons $/ \mathrm{sec} / \mathrm{cm}^{2} /$ steradian $\left(\mathrm{p} / \mathrm{s} / \mathrm{cm}^{2} / \mathrm{sr}\right.$ ). After the IVIS images had been obtained, a reagent (TRI; Sigma-Aldrich) was used to extract RNA from colon tissue samples. The levels of inflammatory gene expression were estimated using a real-time PCR assay (StepOnePlus; Applied Biosystems).

Histopathology examination. Histopathological analysis was used to evaluate C. difficile infection-induced mucosal damage and inflammation. Resected colon tissue samples were fixed in $4 \%$ formaldehyde buffered with PBS and then embedded in paraffin. Deparaffinized $6-\mu \mathrm{m}$-thick sections were stained with hematoxylin and eosin. The results were captured using optical microscopy. Neutrophils were randomly counted in 10 fields for both the spores-alone group and the $\mathrm{Fe}_{3-\delta} \mathrm{O}_{4}$-treated-spores group.

Statistical analysis. GraphPad Prism 5.01 was used for all statistical analyses. All experiments were done in triplicate. Data are means \pm standard error of the mean (SEM) from three independent experiments. One-way analysis of variance (ANOVA) and then Tukey's Multiple Comparison test were used for spore germination curve analyses, and Student's $t$ test was used in the other CFU inhibition test.

\section{References}

1. Morones, J. R. et al. The bactericidal effect of silver nanoparticles. Nanotechnology 16, 2346 (2005).

2. Prucek, R. et al. The targeted antibacterial and antifungal properties of magnetic nanocomposite of iron oxide and silver nanoparticles. Biomaterials 32, 4704-4713 (2011).

3. Jones, N., Ray, B., Ranjit, K. T. \& Manna, A. C. Antibacterial activity of ZnO nanoparticle suspensions on a broad spectrum of microorganisms. FEMS Microbiol Lett 279, 71-76 (2008).

4. Hajipour, M. J. et al. Antibacterial properties of nanoparticles. Trends Biotechnol 30, 499-511 (2012).

5. Barbut, F. et al. Comparison of the efficacy of a hydrogen peroxide dry-mist disinfection system and sodium hypochlorite solution for eradication of Clostridium difficile spores. Infect Control Hosp Epidemiol 30, 507-514 (2009).

6. Hedge, D. D., Strain, J. D., Heins, J. R. \& Farver, D. K. New advances in the treatment of Clostridium difficile infection (CDI). Ther Clin Risk Manag 4, 949 (2008)

7. Voelker, R. I. Clostridium difficile virulence demands new treatment approach. JAMA 303, 2017-2019 (2010).

8. Vardakas, K. Z. et al. Treatment failure and recurrence of Clostridium difficile infection following treatment with vancomycin or metronidazole: a systematic review of the evidence. Int J Antimicrob Agents 40, 1-8 (2012).

9. Loo, V. G. et al. A predominantly clonal multi-institutional outbreak of Clostridium difficile-associated diarrhea with high morbidity and mortality. N Engl J Med 353, 2442-2449 (2005).

10. Jump, R. L., Pultz, M. J. \& Donskey, C. J. Vegetative Clostridium difficile survives in room air on moist surfaces and in gastric contents with reduced acidity: a potential mechanism to explain the association between proton pump inhibitors and $C$. difficile-associated diarrhea? Antimicrob Agents Chemother 51, 2883-2887 (2007).

11. Wilson, K. H. \& Perini, F. Role of competition for nutrients in suppression of Clostridium difficile by the colonic microflora. Infect Immun 56, 2610-2614 (1988).

12. Russell, A. Bacterial resistance to disinfectants: present knowledge and future problems. J Hosp Infect 43, S57-S68 (1999).

13. Owens, R. C., Donskey, C. J., Gaynes, R. P., Loo, V. G. \& Muto, C. A. Antimicrobial-associated risk factors for Clostridium difficile infection. Clin Infect Dis 46, S19-S31 (2008).

14. Burns, D. A., Heap, J. T. \& Minton, N. P. Clostridium difficile spore germination: an update. Res Microbiol 161, 730-734 (2010).

15. Lyras, D. et al. Toxin B is essential for virulence of Clostridium difficile. Nature 458, 1176-1179 (2009).

16. Poutanen, S. M. \& Simor, A. E. Clostridium difficile-associated diarrhea in adults. CMAJ 171, 51-58 (2004).

17. Hirota, S. A. et al. Intrarectal instillation of Clostridium difficile toxin A triggers colonic inflammation and tissue damage: development of a novel and efficient mouse model of Clostridium difficile toxin exposure. Infect Immun 80, 4474-4484 (2012).

18. Ananthakrishnan, A. N. Clostridium difficile infection: epidemiology, risk factors and management. Nat Rev Gastroenterol Hepatol 8, 17-26 (2010).

19. Sebaihia, M. et al. The multidrug-resistant human pathogen Clostridium difficile has a highly mobile, mosaic genome. Nat Genet 38, 779-786 (2006)

20. Wilcox, M. H. \& Fawley, W. N. Hospital disinfectants and spore formation by Clostridium difficile. Lancet 356, 1324 (2000). 
21. Howerton, A., Patra, M. \& Abel-Santos, E. A new strategy for the prevention of Clostridium difficile infection. J Infect Dis 207, 1498-1504 (2013).

22. Howerton, A., Ramirez, N. \& Abel-Santos, E. Mapping interactions between germinants and Clostridium difficile spores. J Bacteriol 193, 274-282 (2011).

23. Xiu, Z.-m, Zhang, Q.-b., Puppala, H. L., Colvin, V. L. \& Alvarez, P. J. Negligible particle-specific antibacterial activity of silver nanoparticles. Nano Lett 12, 4271-4275 (2012).

24. Kumar, A., Pandey, A. K., Singh, S. S., Shanker, R. \& Dhawan, A. Engineered $\mathrm{ZnO}$ and $\mathrm{TiO}_{2}$ nanoparticles induce oxidative stress and DNA damage leading to reduced viability of Escherichia coli. Free Radic Biol Med 51, 1872-1881 (2011).

25. Lee, C. et al. Bactericidal effect of zero-valent iron nanoparticles on Escherichia coli. Environ Sci Technol 42, 4927-4933 (2008).

26. Cavassin, E. D. et al. Comparison of methods to detect the in vitro activity of silver nanoparticles (AgNP) against multidrug resistant bacteria. Journal of nanobiotechnology 13, 1 (2015).

27. Hamal, D. B. et al. A multifunctional biocide/sporocide and photocatalyst based on titanium dioxide $\left(\mathrm{TiO}_{2}\right)$ codoped with silver, carbon, and sulfur. Langmuir 26, 2805-2810 (2009).

28. Gopinath, P. M. et al. Multi-functional nano silver: A novel disruptive and theranostic agent for pathogenic organisms in real-time. Sci Rep 6, 34058 (2016).

29. Jandhyala, S. M. et al. Role of the normal gut microbiota. World J Gastroenterol 21, 8787-8803 (2015).

30. Huang, C. C. et al. Size-control synthesis of structure deficient truncated octahedral $\mathrm{Fe}_{3-\delta} \mathrm{O}_{4}$ nanoparticles: high magnetization magnetites as effective hepatic contrast agents. J Mater Chem 21, 7472-7479 (2011).

31. Li, T. J. et al. In vivo anti-cancer efficacy of magnetite nanocrystal-based system using locoregional hyperthermia combined with 5-fluorouracil chemotherapy. Biomaterials 34, 7873-7883 (2013).

32. Sorg, J. A. \& Sonenshein, A. L. Inhibiting the initiation of Clostridium difficile spore germination using analogs of chenodeoxycholic acid, a bile acid. J Bacteriol 192, 4983-4990 (2010).

33. Lawley, T. D. et al. Use of purified Clostridium difficile spores to facilitate evaluation of health care disinfection regimens. Appl Environ Microbiol 76, 6895-6900 (2010).

34. Kubota, H. et al. Development of TaqMan-based quantitative PCR for sensitive and selective detection of toxigenic Clostridium difficile in human stools. PLoS One 9, e111684 (2014).

35. Lee, N. et al. Iron oxide based nanoparticles for multimodal imaging and magnetoresponsive therapy. Chem Rev 115, 10637-10689 (2015).

36. Laurent, S. et al. Magnetic iron oxide nanoparticles: synthesis, stabilization, vectorization, physicochemical characterizations, and biological applications. Chem Rev 108, 2064-2110 (2008).

37. Yung, P. T. \& Ponce, A. Fast sterility assessment by germinable-endospore biodosimetry. Appl Environ Microbiol 74, 7669-7674 (2008).

38. Wu, Y. N. et al. The anticancer properties of iron core-gold shell nanoparticles in colorectal cancer cells. Int J Nanomedicine 8, 3321 (2013)

39. Levy, S. B. \& Marshall, B. Antibacterial resistance worldwide: causes, challenges and responses. Nat Med 10, S122-S129 (2004).

40. Pelgrift, R. Y. \& Friedman, A. J. Nanotechnology as a therapeutic tool to combat microbial resistance. Adv Drug Deliv Rev 65, 1803-1815 (2013).

41. Balkundi, S. S., Veerabadran, N. G., Eby, D. M., Johnson, G. R. \& Lvov, Y. M. Encapsulation of bacterial spores in nanoorganized polyelectrolyte shells. Langmuir 25, 14011-14016 (2009).

42. Tran, N. et al. Bactericidal effect of iron oxide nanoparticles on Staphylococcus aureus. Int J Nanomedicine 5, 277 (2010).

43. Mariat, D. et al. The Firmicutes/Bacteroidetes ratio of the human microbiota changes with age. BMC Microbiol 9, 123 (2009).

44. Klein, G. Taxonomy, ecology and antibiotic resistance of enterococci from food and the gastro-intestinal tract. Int J Food Microbiol 88, 123-131 (2003).

45. Wexler, H. M. Bacteroides: the good, the bad, and the nitty-gritty. Clinical microbiology reviews 20, 593-621 (2007).

46. Chong, P. M. et al. Proteomic analysis of a NAP1 Clostridium difficile clinical isolate resistant to metronidazole. PLoS ONE 9, e82622 (2014).

47. Lawley, T. D. et al. Antibiotic treatment of Clostridium difficile carrier mice triggers a supershedder state, spore-mediated transmission, and severe disease in immunocompromised hosts. Infect Immun 77, 3661-3669 (2009).

48. Wilcox, M. H. Clostridium difficile infection and pseudomembranous colitis. Best Pract Res Clin Gastroenterol 17, 475-493 (2003).

49. Liu, R., Suárez, J. M., Weisblum, B., Gellman, S. H. \& McBride, S. M. synthetic polymers active against Clostridium difficile vegetative cell growth and spore outgrowth. J Am Chem Soc 136, 14498-14504 (2014).

50. Francis, M. B., Allen, C. A. \& Sorg, J. A. Spore cortex hydrolysis precedes dipicolinic acid release during Clostridium difficile spore germination. J Bacteriol 197, 2276-2283 (2015).

51. Hung, Y. P. et al. Proton pump inhibitor exposure aggravates Clostridium difficile colitis: evidences from a mouse model. J Infect Dis 212, 654-663 (2015).

\section{Acknowledgements}

This work was supported by grants from the Taiwan Ministry of Science and Technology (NSC1022320-B-006-027, NSC101-2314-B-006-048-MY3, and NSC102-2120-M-006- 003); from the Taiwan Ministry of Health and Welfare (MOHW103-TDU-PB-211-113016); from the Taiwan National Research Program for Biopharmaceuticals; and from the Headquarters of University Advancement at National Cheng Kung University, which is sponsored by the Taiwan Ministry of Education. Finally, we thank i-MANI, the International Institute of Macromolecular Analysis and Nanomedicine Innovation, National Cheng Kung University, for providing invaluable technical support.

\section{Author Contributions}

D.-B. Shieh and P.-J. Tsai form the concept, designed the study and edited the manuscript. W.-T. Lee did most experiments and draft the manuscript. Y.-N. Wu helped the organization of the manuscript and perform the primary language editing. Y.-H. Chen participated in most animal tests. S.-R. Wu performed all TEM and cryo-EM imaging and interpretation. T.-M. Shih performed part of the spore germination test. T.-J. Li and L.-X. Yang participated in results discussion and part of the animal tests. C.-S. Yeh provided nanoparticles fabrication protocol and guided the synthesis detail.

\section{Additional Information}

Supplementary information accompanies this paper at doi:10.1038/s41598-017-08387-y

Competing Interests: The authors declare that they have no competing interests. 
Publisher's note: Springer Nature remains neutral with regard to jurisdictional claims in published maps and institutional affiliations.

Open Access This article is licensed under a Creative Commons Attribution 4.0 International License, which permits use, sharing, adaptation, distribution and reproduction in any medium or format, as long as you give appropriate credit to the original author(s) and the source, provide a link to the Creative Commons license, and indicate if changes were made. The images or other third party material in this article are included in the article's Creative Commons license, unless indicated otherwise in a credit line to the material. If material is not included in the article's Creative Commons license and your intended use is not permitted by statutory regulation or exceeds the permitted use, you will need to obtain permission directly from the copyright holder. To view a copy of this license, visit http://creativecommons.org/licenses/by/4.0/.

(c) The Author(s) 2017 